\title{
The Repair and Design Research of the Traditional Residence*
}

\author{
Lili Xiao \\ Xi'an Fanyi University \\ Xi'an, China
}

\begin{abstract}
Dwellings show a regional unique cultural character, and they can reflect the regional human geography in the building. The traditional houses in Guanzhong reflect the unique culture and art of Guanzhong region, and have high artistic value. However, due to the age, a large number of buildings have been destroyed inordinately; this paper discusses the reinforcement and repair design method of brick and wood structure, to find a suitable design method of repairing traditional houses.
\end{abstract}

Keywords-traditional houses; Guanzhong region; cultural inheritance; building design; reinforcement and repair

\section{THE CHARACTERISTICS, VALUE AND STATUS QUO OF TRADITIONAL HOUSES IN GUANZHONG}

Shaanxi Guanzhong area now retains more ancient houses in the Ming and Qing Dynasties. These houses gather in the form of villages. Due to the unique climatic environment, geographical conditions, building materials and living habits of Guanzhong in Shaanxi, the local characteristics of Guanzhong traditional houses are created. Guanzhong traditional houses are in the form of traditional Chinese courtyard, built a concierge, courtyard, principal room, wing-rooms and backyard. Guanzhong residential roof is in the form of hard hillside roof; the wooden frame as the main structural system, to bear the weight of the roof; there are adobe walls, rammed earth walls and brick walls, to bear their own weight.

Affected by the local overall decoration habits, the exquisite wood carvings, brick and stone carvings generally showed under the doors and windows, beams and eaves of Guanzhong houses, and these art components, with unique local features, fully embodies the customs and highlights Guanzhong cultural deposits in Guanzhong region. There are also some courtyards with a beautiful festoon gate or others, and they have a higher artistic value. The existing traditional houses in Guanzhong mostly are Ming and Qing buildings, with a higher historical and cultural value.

*Article Subject Source: Shaanxi Provincial Department of Culture ar subject "Guanzhong traditional houses protection and reinforcement design research", subject number 2015SY057.
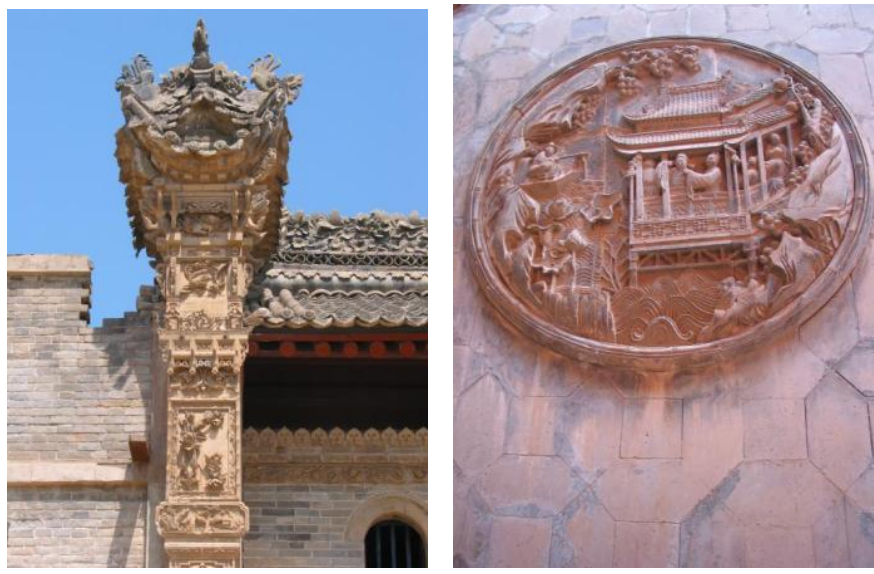

Fig. 1. Tang family courtyard exquisite brick art.

The representative traditional houses of Shaanxi Guanzhong are mainly the Dangjia Village houses, Hancheng ancient city houses, Tang's courtyard, Zhou's courtyard, Duan's courtyard, Gao's courtyard and so on. Most of these buildings were built in the Ming and Qing Dynasties, the Tang's courtyard, Zhou's courtyard and Gao's courtyard are only for the visit, and there are still some residents living in the Dangjia Village, Hancheng ancient city and Duan's courtyard.

Today, the study and protection of traditional houses is in full swing, Shaanxi Guanzhong traditional houses have been generally received extensive attention and protected completely, but due to these houses are so old, they have been broken badly. Wood frame as a load-bearing structure, because of its poor durability and the long load-bearing period, wooden pillars began to appear a variety of different forms of damage, such as cracking, decay, deformation, tenon pulling out, damaged by worms and so on; the wall combined with brick and soil will peel, crack or even collapse after the long-time weathering. Such as "Fig. 2", "Fig. 3", "Fig. 4" and "Fig. 5". In addition, the structure of the traditional houses in Guanzhong is the masonry structure, there are no ring beams and constructional columns, and so seismic performance is poor. Many dwellings even still are used in living, but the strength of buildings that the residents repaired cannot reach the desired effect on and the appearance of the buildings had changed obviously, undermining the original form and coordination of the traditional houses. 


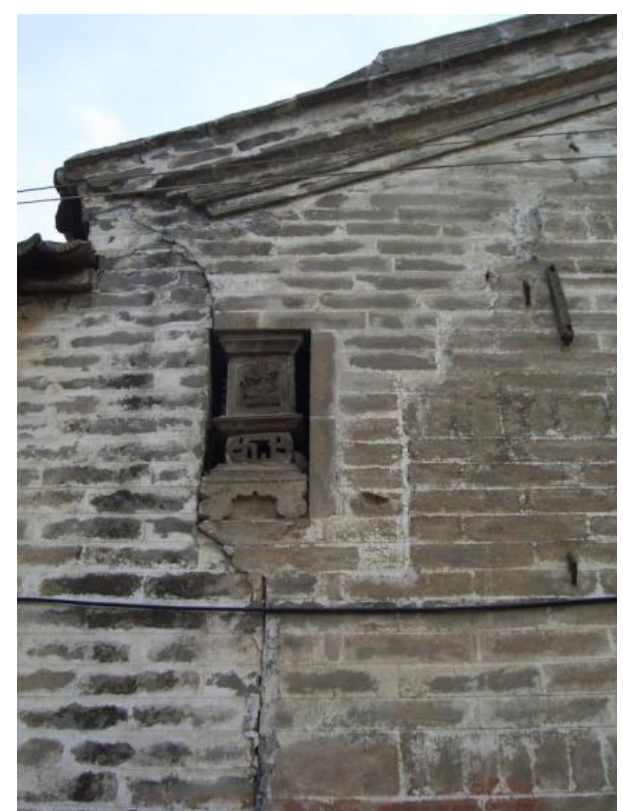

Fig. 2. Jiulangmiao Lane dwelling wall cracking, Hancheng ancient city

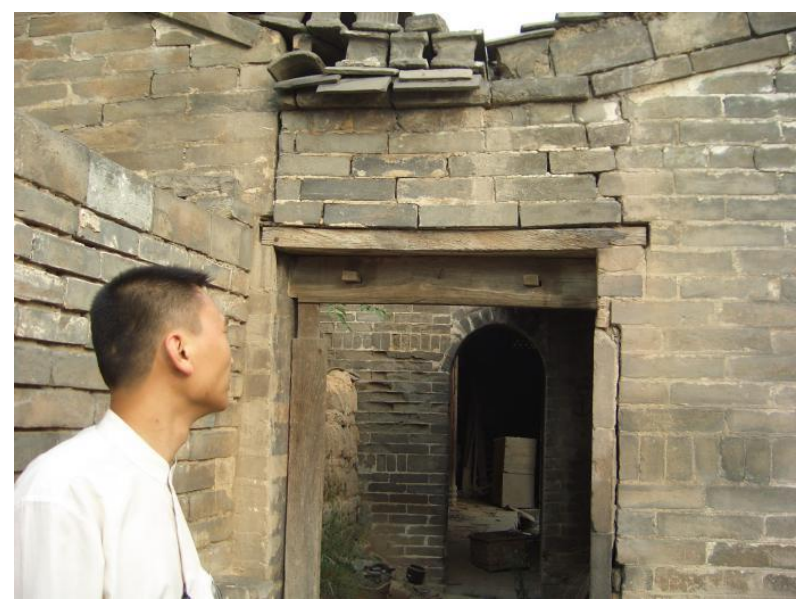

Fig. 3. Dangjia Village wall cracking, wood lintel deflection is too large

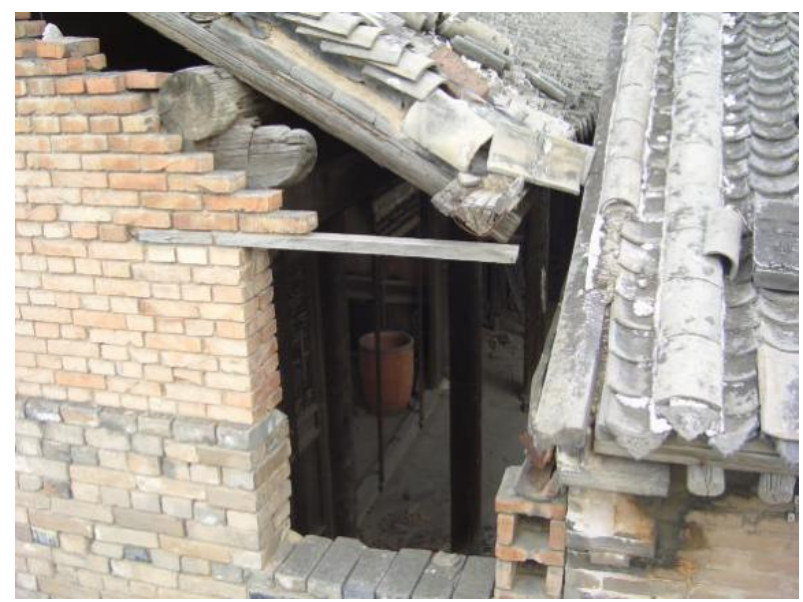

Fig. 4. Dangjia Village wooden frame damage, the red brick wall repaired by the resident is not coordinated

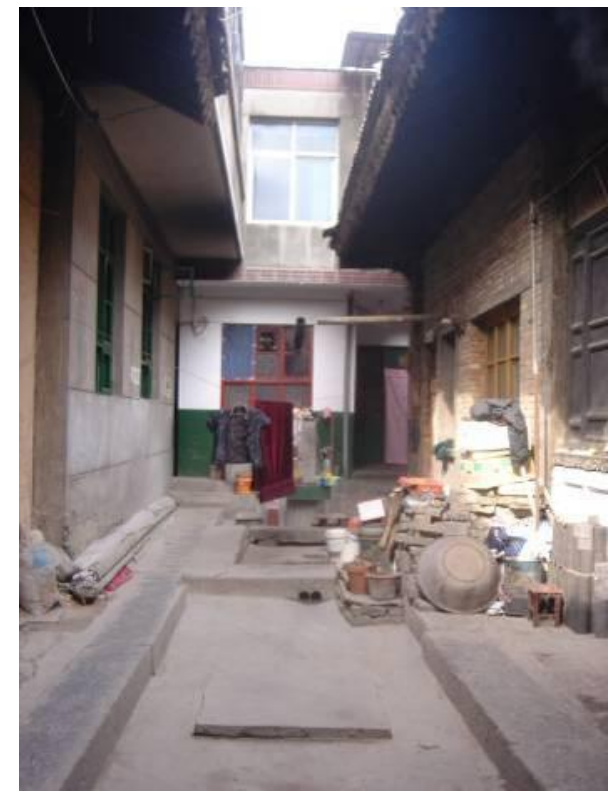

Fig. 5. Duanjia's Courtyard repaired and transform by the villagers

\section{DESIGN METHOD OF TRADITIONAL DWELLING HOUSES REPAIR}

In China, the traditional building repair design has always complied with the principle of "remain old as before" advocated by Mr. Liang Sicheng, and the "Ancient Building Wood Structure Maintenance and Reinforcement Technical Specifications", promulgated in 1992, also provides that the basic principle of repairing ancient buildings is not to change the original shape of the building, pay attention to the preservation of the original architectural form, building structure, building materials, arts and crafts [12].

\section{A. Repair Design For Wood Frame Damage}

Guanzhong traditional houses use wooden pillars and wooden beams as the main load-bearing components, but because of insects, weathering, decay, long-time load and other reasons, leading to the wood components bending, cracking and even breakage, and the frequently-used reinforcement methods are chemical reinforcement method, structural strengthening method and carbon fiber sheet reinforcement method.

\section{1) Chemical reinforcement method}

Chemical reinforcement method is the use of chemical substances to deal with wood to stabilize it, not only can improve the strength of wood components, but also improve the anti-corrosion and insect resistance and dimensional stability. The frequently-used chemical reinforcement agents are inorganic compounds and organic compounds, the former is mainly used for the protection of wet wood, and the latter is mainly used for the protection of wood that the moisture content above the fiber saturation point. The curing methods of chemical agent are vacuum method, thermo catalytic curing method, photochemical method, freeze-drying method and so on. [8] 


\section{2) Structural strengthening method}

a) Clamping connection

Mainly used in the wooden beam of wall damaged by the worms, corrosion and others, when the length of damaged beam is greater than the $1 / 3$ length of beam or the head of beam is damaged by worms completely, it must use the clamping connection method to strengthen [8], as shown in "Fig. 6".

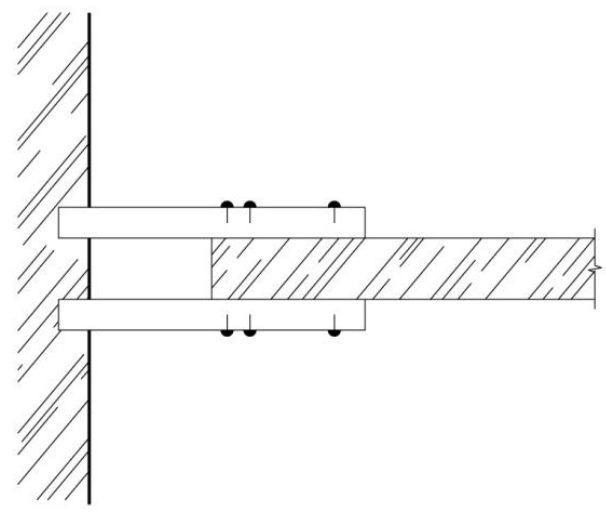

Fig. 6. Wooden beams clamping reinforcement.

b) Supporting connection

Use the box iron and other materials under the beam to support the beam, as shown in "Fig. 7".

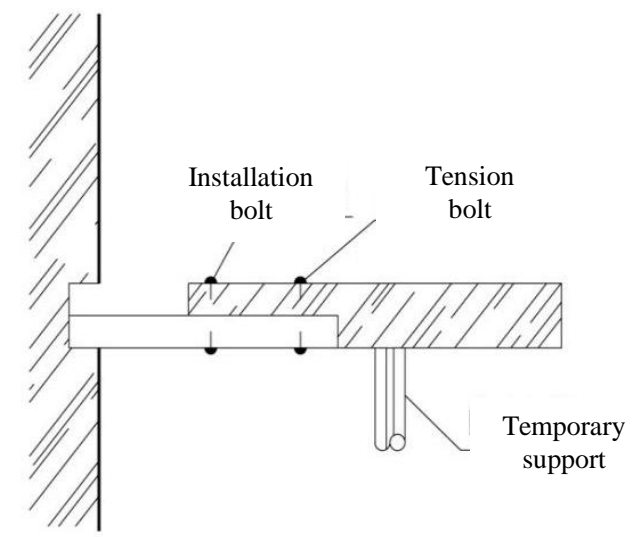

Fig. 7. Wooden beams supporting reinforcement.

\section{c) Flat steel reinforcement}

Flat steel reinforcement method is generally used for wood beam longitudinal splitting damage, the construction requirements is to ensure that the bolt tightening fixed, so that the flat steel without loosening, the beam surface cracks should be filled, as shown in "Fig. 8".

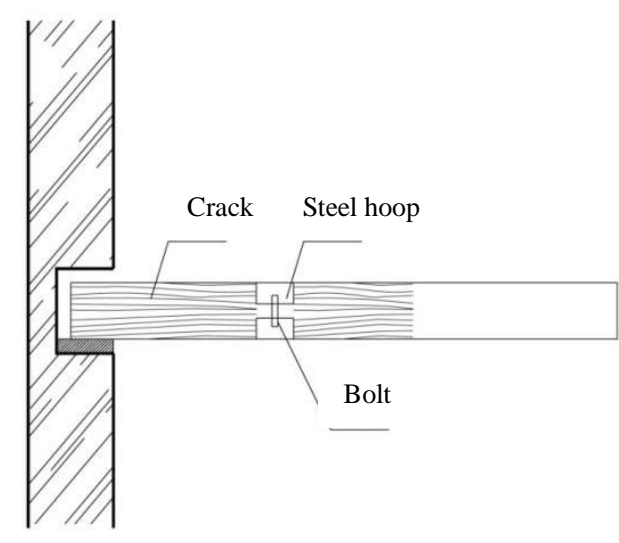

Fig. 8. Flat steel reinforcement.

d) Diagonal bracing double - clamp method

Construction requirements: according to the design requirements to cut materials, plywood should be parallel symmetrical installation, and connected with beam column closely. joints

e) Supporting wood reinforcement of beam and column

Supporting wood reinforcement: setting load bearing wood at the end of the damaged beam, supporting wood and the column are closely connected by bolts in line with the construction requirements, as shown in "Fig. 9".

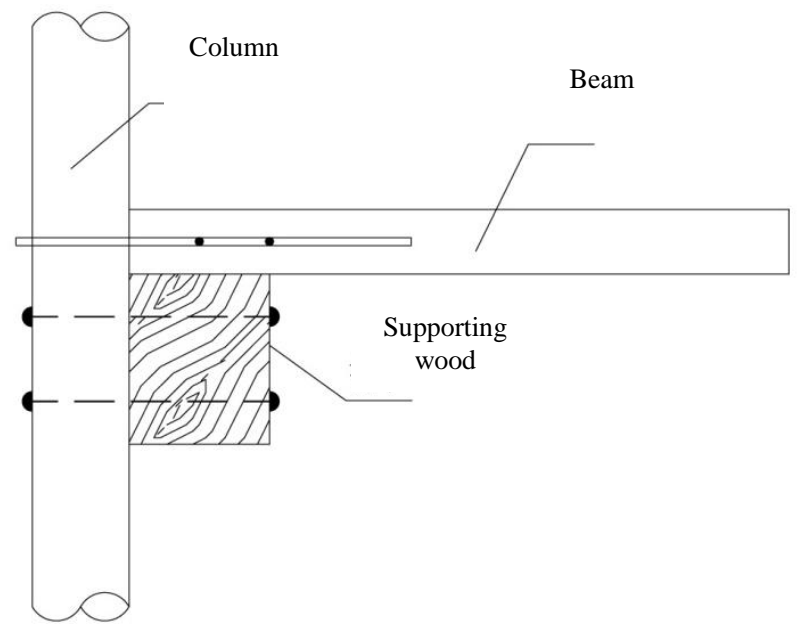

Fig. 9. Supporting wood reinforcement of beam and column joints.

\section{3) Carbon fiber sheet reinforcement method}

Carbon fiber is a microcrystalline graphite material obtained by high temperature firing of organic fibers, with the characters of light weight, high strength, high modulus, good corrosion resistance, good fatigue resistance and good design ability. Sticking wood components and joints with carbon fiber sheet can improve the bearing capacity, stiffness and seismic performance of wood structure. In recent years, with the carbon fiber sheet material prices dropped significantly, its application 
in the construction has increased rapidly, so it has gradually increased in the wood structure repair and reinforcement.

\section{REPAIR DESIGN FOR WALL DAMAGE}

Guanzhong traditional houses in the widespread use of brick masonry walls, the wall does not bear the weight of the roof, only bear their own load and play a role in enclosure. According to the Dangjia Village, Hancheng ancient city and other research, and measured with the rebound method, we found that brick walls and mortar joint strength are not high, and a large number of walls have the vertical cracks and oblique cracks, and some wall's green bricks are exfoliated or even collapsed. Existing wall reinforcement methods are cement grouting or spray repair method, steel mesh cement mortar surface reinforcement method, spray glass fiber polymer method and so on.

\section{A. Cement Grouting or Spray Repair Method}

Cement grouting is to press the cement into the brick wall cracks through the pressure equipment, to make them bonded. The spray repair method is the use of compressed air to spray the cement mortar or fine aggregate concrete to the surface, solidifying into a new spray layer [2], and mainly used to increase the brick walls, columns and other structural components section. The disadvantage of this method is the need for specialized equipment. For the repair of traditional houses, compared to the latter, cement grouting method can repair the wall cracks well without changing the face of the wall, and it is more desirable. But when it used alone, it can improve the wall bearing capacity significantly.

\section{B. Steel Mesh Cement Mortar Surface Reinforcement Method}

Steel mesh cement mortar surface reinforcement method is to add a layer of steel mesh on the surface of the brick wall, before fixing steel mesh, it need to clean up the wall surface decoration layer, and then anchor steel bar, and then plaster the steel mesh with cement mortar or fine stone concrete. Generally plaster both sides of the steel mesh when reinforcement, so this method is also called "plywood wall" reinforcement method. This method can significantly improve the bearing capacity of the wall, resist lateral stiffness and wall ductility, the structural performance of normal service stage has also been improved [9], so ordinary brick wall repair more use of this method. But because of the larger changes in the face of the wall, the traditional residential wall repair carefully used.

\section{Expanded Section Reinforcement Method}

Expanded section reinforcement method is to increase the cross-sectional area of the brick wall to improve the bearing capacity of the original wall, but also can improve the rigidity of its cross-section, enhance the stability and seismic capacity of the structure, so that improve the performance required by the normal use. However, this method needs a lot of wet construction on the site, the construction period is long, and the original structure will be affected. It is mainly used in the masonry bearing capacity is insufficient, but the masonry has not yet cracked or only a slight crack, and the independent brick column, brick pilaster, wall between window that required expansion of the cross-sectional area and other loadbearing walls with insufficient bearing capacity.

\section{Structural Steel Wrapping Reinforcement Method}

Structural steel wrapping reinforcement method firstly need to remove the paint layer around of the wall and wash it clean, and plaster a $10 \mathrm{~mm}$ thick leveling layer with cement mortar in the surface of corner, then paste the structural steel in the corner of the wall, chucking with a fixture, and then connect all the structural steel through the batten plate, finally remove the fixture and render cement mortar to protect the structural steel. Because the width of the wall is much larger than the thickness, only using four structural steel in corners cannot effectively constrain the middle of the wall, so it should add a slab in vertical direction on both sides of the middle of the wall, and binding with bolts, finally, plastering the mortar protection layer [4]. This method is simple to construct, the field workload and wet work are less, the bearing capacity improving obviously, apply to the brick wall that not allowed to expend the cross-sectional size, but requiring improving its bearing capacity, while the method only has a little change for the wall's appearance [7].

\section{E. Supporting Beam and Changing Column}

Supporting beam and changing column is mainly used for the bearing capacity of the independent brick column is seriously insufficient, setting a temporary support and unloading of the brick column load, and then determining the material strength and the cross-sectional dimension of the new brick column according to the calculation and setting beam pad under the girder[1]. And the joist adding column is mainly used for the bearing capacity of the wall between window is seriously insufficient, the first set of temporary support, and then considering the new reinforced concrete column bearing the all load and calculating the cross section and reinforcement of the added column. Construction need to remove some of the original brick wall, then twisted into a zigzag shape, and then tied steel bars, supporting template and pouring concrete [11]. This method is mainly used for brick masonry bearing capacity is seriously inadequate, masonry fragmentation even collapse [4].

\section{F. Spray Glass Fiber Polymer Method}

Glass fiber reinforced polymer material has light, high strength, convenient construction, good durability and other characteristics, spray glass fiber polymer reinforcement method is to short glass fiber and high performance adhesive mixed slurry [4], with injection machinery, rapidly spray it to the bearing surface, to improve the bending strength and shear strength of the structural components, and improve the ductility and toughness of the component [11]. The glass fiber is light and has high strength, the additional volume produced by the reinforcement repair is small, and it will not change the appearance of the structure of the reinforced building, so it is beneficial for the traditional residential repair.

\section{THE REPAIR DESIGN FOR THE OVERALL IMPROVEMENT}

Guanzhong traditional houses generally do not set the ring beam and structural columns, so the building integrity and 
seismic performance are poor. Adding the ring beam and the structural column shall provide a circle of continuous ring beams at the top of the building wall and structural columns at the four corners of the building. The lower part of the vertical steel bar shall be fixed to the 0.5 meter under the ground, the upper part shall be connected with the ring beam and the steel bar shall be provided with stirrups, and the steel tie bar shall be arranged every half meter. The structural column shall be partially removed from the wall, forming a zigzag, then supporting template pouring concrete, so that ring beam, structural column and wall are connected into a whole. This method can improve the seismic performance of brick masonry building, the construction is simple, the technology is mature, and the appearance of the building is not changed, suitable for the traditional houses reinforcement repair.

\section{CONCLUSION}

The repair design of Guanzhong traditional houses brick and wood construction should consider repairing damaged structure, improving the carrying capacity and durability, and also consider the protection of the appearance of the building, cannot change it obviously. And the changed point can be convenient to recognize to make the descendants to distinguish the original building structure and the added components. In view of the above requirements and combined with the above repair methods, the conclusion is that Guanzhong traditional houses reinforcement should first add the ring beam and structural columns, and for the wall that its bearing capacity is not high, can use of cement grouting method to repair, and for the wall that its bearing capacity is high, can use of spraying glass fiber polymer method to repair. And for the wooden frame beam and column repair, can use of the above several methods.

\section{REFERENCES}

[1] He Jian, Masonry Structure House Reliability Identification and Reinforcement Methods and Examples [D], Chongqing University, 2007

[2] Wu Yankun, Existing Building Structure Wind Resistance Identification and Reinforcement [D], Tongji University, 2008

[3] Wu Xin, Study on the Ecological Design of Modern Courtyard House in Guanzhong [D], Chongqing University, 2009

[4] Wang Yonghu, Finite Element Analysis of Spinning GFRP Shear Reinforcement without Reinforcement [D], Wuhan University of Technology, 2009

[5] Chen Wei, Hu Bin, a Few of Views on the Use of Cultural Relics Repair Principle [J], new building, 2006(5): 6-8.

[6] Li Gang. Study on the Modern Adaptive Transformation of Guanzhong Dwellings [D], Xi'an University Of Architecture and Technology, 2007.

[7] Xu Yan. Study on the Model of Traditional Residential Architecture in Guanzhong [D], Xi'an University of Technology, 2010.

[8] Chen Juan. Finite Element Analysis of Block Rib Reinforced with the Mechanical Effect of Ancient Building [D], Southwest Petroleum University, 2013

[9] Fan Xiaodong, Analysis of Bearing Capacity and Seismic Performance of Existing Masonry Structures [D], Chongqing University, 2013

[10] Gao Wei, Inheritance and Development of the Characteristics of Guanzhong dwellings in Modern Architecture of Xi'an [D], Xi'an University of Architecture and Technology, 2012.

[11] Liu Li, Study on the Shear Behavior of Unreinforced FRP Masonry Walls [D], Wuhan University of Technology, 2009.
[12] Meng Tao, Study on the Failure Mechanism and Reinforcement Simulation of Mortise and Tenon Joints of Wood Structure Ancient Buildings in Huizhou [D], Anhui University of Architecture, 2013.

[13] Zhao Pengtu. Discussion on Measures for Strengthening and Repairing of Traditional Houses Base Reinforcement [J], Shanxi Architecture, 2014 (20): 83-84.

[14] Zhu Jinbiao. The Carbon Fiber Reinforced Plastics Reinforcement and Repair of Damaged Wood Beams (CFRP) Test Research [D]. Zhejiang University, 2005. 